\title{
AUXILIANDO O PROCESSO DECISÓRIO NA AGRICULTURA 4.0: REVISANDO OS MÉTODOS ORDINAIS NA SELEÇÃO DE DRONES
}

\author{
Heider Silva Barros \\ Centro de Tecnologia da indústria Química e Têxtil (SENAI CETIQT) \\ Rua Magalhães Castro 174 - Riachuelo - Rio de Janeiro - RJ - Brasil \\ barrosheider72@gmail.com \\ Marcone Freitas dos Reis \\ Centro de Tecnologia da indústria Química e Têxtil (SENAI CETIQT) \\ Rua Magalhães Castro 174 - Riachuelo - Rio de Janeiro - RJ - Brasil \\ marconefreis11@gmail.com \\ Marcos dos Santos \\ Instituto Militar de Engenharia (IME) \\ Praça General Tibúrcio, 80 - Praia Vermelha - Urca - Rio de Janeiro - RJ \\ marcosdossantos_doutorado_uff@yahoo.com.br \\ Kaique de Oliveira Dutton da Silva \\ Centro de Tecnologia da indústria Química e têxtil (SENAI CETIQT) \\ Rua Magalhães Castro 174 - Riachuelo - Rio de Janeiro - RJ - Brasil \\ kaiquedutton@gmail.com \\ Valéria da Silva Brito \\ Universidade Federal Fluminense (UFF) \\ Av. Visconde do Rio Branco, s/n - Centro, Niterói - RJ, 24020-140 \\ valeriasbrito@hotmail.com
}

\begin{abstract}
RESUMO
A agricultura 4.0, derivada da Indústria 4.0, é um fenômeno além da mecanização do campo. Adotando-se recursos computacionais de alto nível tecnológico, sensores, comunicação entre máquinas (M2M), técnicas de análise e conectividade entre dispositivos móveis as operações no campo passaram a ser orientadas e a processar um enorme volume de dados que servirão de base para a tomada de decisões. A gama de tecnologias tornaram a agricultura 4.0 um divisor de águas no setor. O benefício mais básico dessas novas ferramentas é o aumento da produtividade. Aliando-se a utilização de drones a agricultura 4.0 é possível se atingir altos níveis de redução de custos, economia do tempo, mão de obra e estoques, maior precisão e eficiência. Dentro desse contexto, o objetivo do presente estudo é ranquear modelos de drones disponíveis no mercado aplicando os métodos ordinais de Borda, Condorcet e Copeland, e pretende-se a partir do ranqueamento proporcionar ao decisor um norte em suas alternativas.
\end{abstract}

Palavra-chave: Agricultura 4.0; Método Borda; Método Condorcert; Método Copeland; Drones. 


\begin{abstract}
Agriculture 4.0, derived from Industry 4.0, is a phenomenon beyond the mechanization of the field. Adopting high-tech computing resources, sensors, machine-tomachine communication (M2M), analytical techniques, and mobile device connectivity, field operations are now being driven and processing a huge amount of data that will serve as the basis for taking of decisions. The range of technologies has made agriculture 4.0 a watershed in the industry. The most basic benefit of these new tools is increased productivity. By combining the use of drones with agriculture 4.0 its is possible to achieve high levels of cost savings, time savings, labor and inventory, greater accuracy and efficiency. Within this context, the objective of the present study is to rank commercially available drone models by applying the Ordinal Borda, Condorcet and Copeland methods, and it is intended from the ranking to provide the decision maker with a north in his alternatives.
\end{abstract}

Keywords: Agriculture 4.0; Borda Method; Condorcert Method; Copeland Method; Drones.

\title{
Como Citar:
}

BARROS, Heider Silva; REIS, Marcone Freitas dos, SANTOS, Marcos dos; SILVA, Kaique de Oliveira Dutton da; BRITO, Valéria da Silva. Auxiliando o Processo Decisório na Agricultura 4.0: Revisando os Métodos Ordinais na Seleção de Drones. In: SIMPÓSIO DE PESQUISA OPERACIONAL E LOGÍSTICA DA MARINHA, 19., 2019, Rio de Janeiro, RJ. Anais [...]. Rio de Janeiro: Centro de Análises de Sistemas Navais, 2019.

\section{INTRODUÇÃO}

A tecnologia empregada no campo foi determinante para que a agricultura brasileira alcançasse o patamar atual. A evolução é contínua e agora se consolida uma nova era de tecnologia agrícola. Hoje já não existe mais separação entre os mundos físico e virtual, conectados para facilitar a vida das pessoas. Por trás dessa ideia está o conceito da Agricultura 4.0 (Agro 4.0), também chamada de agricultura digital, uma clara referência à Indústria 4.0, inovação que teve início na indústria automobilística alemã e que agora conquista fábricas de diversos segmentos devido à completa automatização proporcionada aos processos produtivos. (VDMA VERLAG, 2016)

De acordo com Massruhá; Leite (2014), a Agro 4.0 emprega métodos computacionais de alto desempenho, rede de sensores, comunicação máquina para máquina (M2M), conectividade entre dispositivos móveis, computação em nuvem, métodos e soluções analíticas para processar grandes volumes de dados e construir sistemas de suporte à tomada de decisões de manejo. Além disso, contribuirá para elevar os índices de produtividade, da eficiência do uso de insumos, da redução de custos com mão de obra, melhorar a qualidade do trabalho e a segurança dos trabalhadores e diminuir os impactos ao meio ambiente. Engloba a agricultura e pecuária de precisão, a automação e a robótica agrícola, além de técnicas de big data e a Internet das Coisas.

A agricultura é a atividade econômica mais dependente das condições climáticas. Além de influenciar o crescimento, o desenvolvimento e a produtividade das culturas, o clima afeta também a relação das plantas com insetos e microrganismos, favorecendo ou não a ocorrência de pragas e doenças. O monitoramento agrometeorologico consiste na coleta sistemática e contínua de dados meteorológicos para a produção de informações de interesse ou uso agrícola. Sistemas que integram de forma coordenada e simultânea as funções de 
coleta, transmissão e processamento de dados podem fornecer informações agrometeorologias atualizadas em tempo quase real. Existem várias práticas agrícolas que podem se beneficiar de informações agrometeorologias, destacando-se: o preparo do solo, a semeadura, a adubação, a irrigação, o controle fitossanitário, a colheita etc. Estimativas de produtividade, de qualidade da produção e de favorabilidade à ocorrência de doenças também necessitam de dados meteorológicos. (MONTEIRO; OLIVEIRA, NAKAI, 2014)

Nesse cenário, um novo método de precisão agrícola que vem sendo utilizado na agricultura, são os drones e/ou VANTs (Veículos Aéreos Não Tripulados), tal utilização se fortaleceu pelos recentes avanços na tecnologia da computação e desenvolvimento de sistemas globais de navegação e geoprocessamento. Estas "aeronaves" portam sensores e recursos de imagem eficientes e precisos, colaborando com danos na lavoura e índice de crescimento, por exemplo. (ANDRADE, 2016)

Oliveira et al. (2016), colocam que a procura por procedimentos tecnológicos em função da produção agrícola limpa gera a procura de novos desenvolvimentos e novos métodos no setor, objetivando a eficiência do plantio e a redução dos impactos ambientais na produção. Em 2017, o Governo Brasileiro com apoio da União Europeia realizou um projeto de estudo sobre a indústria de veículos aéreos não tripulados e concluíram que o equipamento pode e deve ser utilizado na agricultura do futuro. Para todos os países envolvido com o estudo, também entendem que o consumo da agricultura tem aumentado exponencialmente e aumentará nos próximos anos, devido ao crescimento da população mundial (Ministério da Indústria, Comércio Exterior e Serviços, 2017).

Dentro desse escopo o presente estudo tem por objetivo auxiliar o processo de tomada de decisão na seleção de drone através da aplicação dos métodos ordinais Borda, Condorcet e Copeland e oferecer um norte ao decisor a partir do rankeamento de alternativas gerados pelos métodos.

\section{DESCRIÇÃO DO PROBLEMA}

Diante dos desafios apresentados na agricultura, principalmente o de aumentar a produção agrícola sem ampliar a área plantada significativamente, surgem novas oportunidades para a utilização de inovações na área de TIC. As tendências apontam que o setor agropecuário demandará novas TICs para gestão de dados, informações e conhecimentos em todas as etapas da cadeia produtiva em uma nova infraestrutura onde os mundos físico e digital estão totalmente interconectados. (MASSHRUÁ, 2015)

Segundo o governo brasileiro e o Europeu, até 2050 haverá aumento da produção agrícola, devido ao crescimento da população mundial e isso a agricultura deverá acompanhar a demanda evitando danos ambientais, sendo mais sustentáveis. As supervisões de safra na agricultura, utilizada com imagens de satélite são muito caras e imprecisas para o agricultor moderno e o Drone, oferece o monitoramento da safra por um custo muito menor, integrando todas as fases de vida de uma plantação.

Métodos de apoio multicritério à decisão (AMD) têm sido empregados em diferentes áreas do conhecimento (RODRIGUEZ et al., 2013). Tais métodos ajudam na tomada de decisão na qual haja avaliação de alternativas em múltiplas dimensões com base nas preferências emitidas pelos agentes de decisão (GOMES et al.,2004; HAIMES E CHANKONG, 1979).

Utilizar as ferramentas de apoio a decisão como aliada da agricultura 4.0, pode capacitar o agricultor/produtor a analisar as alternativas (novas tecnologias), em múltiplos pontos de vista em busca de uma solução satisfatória para o negócio.

A partir do assunto em tela, confeccionou-se o mapa mental apresentado na Figura 1 a seguir, a fim de facilitar a visualização das condições de contorno do problema. 
Figura 1 - Mapa Mental

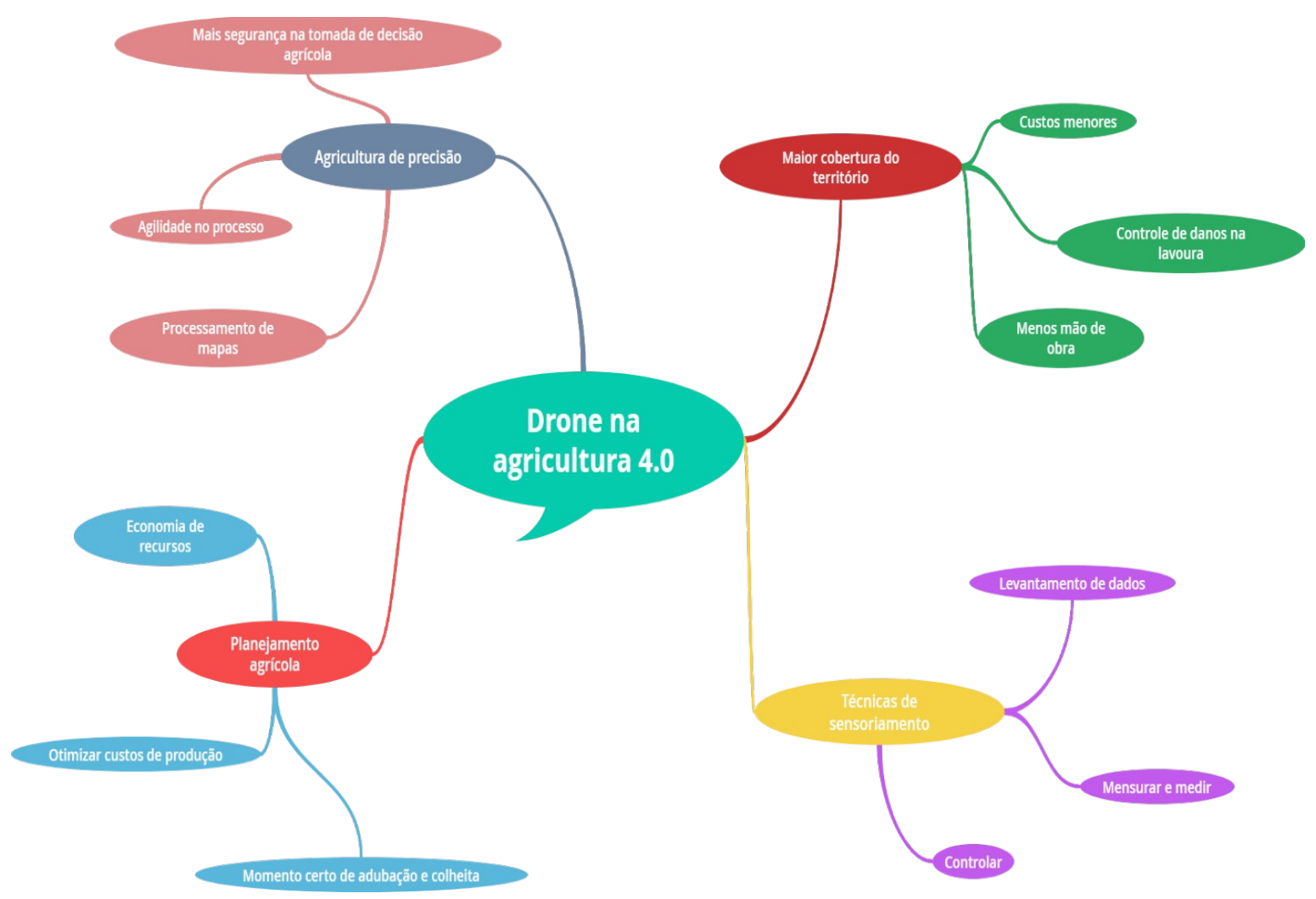

Fonte: Autor (2019)

\section{FUNDAMENTAÇÃO TEÓRICA}

\subsection{Agricultura 4.0}

A Agricultura 4.0 é o aproveitamento dos avanços nas tecnologias da informação e da comunicação (TICs) na agricultura como forma de repensar e redesenhar processos ao longo de toda a cadeia de valor, do campo à mesa, abrindo possibilidades para a geração de uma vasta gama de inovações para o mundo da agricultura e da alimentação. O termo Agricultura 4.0 foi criado pela revolução da transformação digital, que substitui átomos (mundo físico) por bits (mundo digital), transformando itens físicos em bits. Na prática, o que se quer é que a agricultura possa acessar uma vasta gama de inovações baseadas, por exemplo, em sensores capazes de fornecer dados cada vez mais precisos, visualização e previsões de condições meteorológicas para melhor gestão das lavouras; monitoramento autônomo e intervenções precisas nos processos de gestão da produção agropecuária; comunicação altamente integrada e automação das mais variadas atividades nos setores agroalimentar e agroindustrial; sistemas avançados de monitoramento, rastreabilidade e controle que informem e assegurem aos consumidores sobre segurança e sustentabilidade dos alimentos, dentre muitas outras inovações e avanços. (AGRISHOW, 2016)

O Brasil já se destaca na chamada Agricultura 4.0, em especial pela incorporação de processos. Dentre as melhorias incorporadas estão práticas e processos de precisão, amplo uso de sensores e mecanismos sofisticados de previsão e resposta a variações de clima, além de abrir espaços para o Brasil em segmentos estratégicos da agricultura e da bioeconomia, economia sustentável baseada em recursos biológicos e processos limpos e renováveis. Big 
Data, inovação, drones, IoT (Internet das Coisas), veículos autônomos, transformação digital, robótica, todas estas expressões já fazem parte do vocabulário cotidiano dos novos "homens do campo", os produtores e trabalhadores rurais que estão vivendo e promovendo a revolução tecnológica do agronegócio. (AGRISHOW, 2016)

\subsection{AgRiCUltURA DE PRECISÃo E O USO DA TECNOLOGIA PARA SUSTENTABILIDAdE AGRÍCOLA}

Umas das definições mais importantes e completas conceitua a Agricultura de Precisão como uma nova forma de gestão ou de gerenciamento agrícola, fazendo parte desse modelo um conjunto de tecnologias e procedimentos utilizados para que as lavouras e os sistemas agrícolas sejam otimizados, tendo como elemento-chave o gerenciamento da variabilidade espacial e temporal da produção e dos fatores associados. (SWINTON; LOWENBERG-DEBOER, 1998)

A Agricultura de Precisão é considerada como uma estratégia de gestão que utiliza as tecnologias da informação para trazer os dados de múltiplas fontes e apoiar as decisões relacionadas com a produção vegetal. A Agricultura de Precisão tem três componentes: captação de dados em uma escala e frequência adequada, interpretação e análise desses dados, gestão e implementação de uma resposta a uma escala espacial e de tempo adequada. É provável que o impacto mais significativo da Agricultura de Precisão ocorra na forma como as decisões de gestão da variabilidade espacial e temporal no sistema produtivo vegetal serão tomadas. (INAMASU et al., 2011)

Na Figura 2 a seguir, são apresentadas as etapas da Agricultura de Precisão.

Figura 2 - Ciclo da Agricultura de Precisão

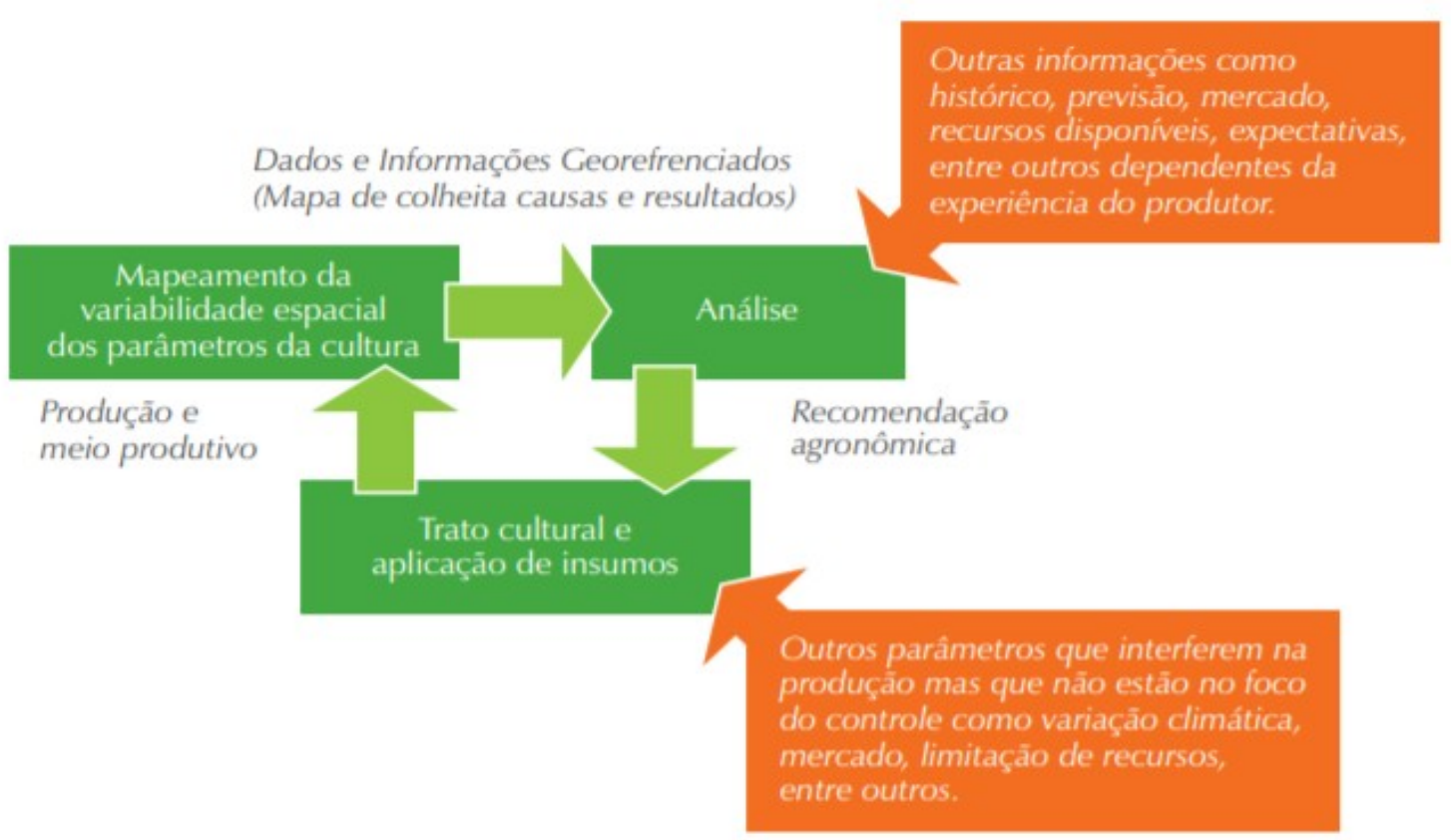

Fonte: INAMASU et al. (2011)

Segunda a EMBRAPA (2012), a agricultura do futuro deverá estar balizada por conceitos, métodos e aplicabilidades multifuncionais, muito além da visão convencional, da agricultura dedicada à produção de alimentos, fibras e energia. Os padrões tecnológicos do agronegócio mundial já estão sendo alterados pela introdução de novas tecnologias resultantes de avanços muito recentes do conhecimento científico. Sustentada em tais avanços e sintonizada com a emergente Economia Verde, a agricultura deverá se nortear por 
um novo conjunto de funcionalidades e requisitos, como os exemplificados na figura abaixo, que deverão conformar o padrão tecnológico do agronegócio do futuro.

Estes novos requisitos e funcionalidades delineiam um agronegócio organizado em torno de vertentes tecnológicas voltadas para a consolidação de sistemas agroalimentares e agroindustriais limpos, com balanço positivo de carbono, que integre qualitativamente a relação campo-cidade, com cadeias e arranjos calcados na sustentabilidade e na inclusão produtiva, principalmente dos agricultores familiares e pequenos produtores. (EMBRAPA, 2012)

\subsection{DRONES: Conceito E APLiCAÇão nA AGRICUltura}

Veículos aéreos não tripulados (VANTs/Drones), como o nome sugere, são aeronaves que decolam e voam sem a necessidade de tripulação a bordo. O controle das atividades comumente executadas pelos tripulantes pode ser executado por sistemas computacionais programados e/ou à distância por seres humanos via controles remotos. (EID et al., 2013)

Atualmente, no Brasil já se pode falar no uso de Drones gerando imagens importantes para a agricultura de precisão. O monitoramento de safras a partir de imagens possibilita a aquisição de dados da área das lavouras, desde a fase do plantio até a fase da colheita. Tais informações são úteis para o manejo e monitoramento de safras, bem como na gestão e logística da produção, entre outros. (SILVA NETO, 2013)

Honkavaara et al., (2013) afirmam que os métodos que se utilizam de Drones possibilitam a coleta de dados eficientes, em termos de custo, com o espaço desejado e resoluções temporais. Para os autores, uma importante vantagem dessa tecnologia se refere ao fato que, os dados de sensoriamento remoto podem ser obtidos ainda que sob condições de imagem pobres, ou seja, sob a cobertura de nuvens, fato que torna o método operacional em uma ampla gama de aplicações de medição ambiental.

Os Drones na agricultura são utilizados em situações diversas como, por exemplo, para detecção e controle de invasores na plantação de milho. Uma grande vantagem desse tipo de uso, é que os Drones podem operar a altitudes mais baixas e, portanto, a captura de imagens com uma resolução espacial muito elevada (de alguns centímetros ou milímetros), o que não seria viável com planos convencionais ou satélites. Isso é fundamental para discriminar entre as pequenas mudas de plantas daninhas e de culturas em estágios iniciais, na maioria dos campos. (PEÑA-BARRAGÁN et al., 2012)

\subsection{A Pesquisa Operacional e os Métodos Multicritério no Apoio a Decisão}

Segundo Arenales (2007), pesquisa operacional é um enfoque científico sobre a tomada de decisões. Para alguns autores, pesquisa operacional significa as decisões que são adotadas, para melhorar o funcionamento de um sistema levando em conta os recursos da empresa. Assim, a pesquisa operacional busca a decisão ótima para um problema.

As tarefas de avaliar e selecionar fornecedores envolvem diversos critérios no processo de tomada de decisão e reconhece-se que se trata, inerentemente, de um problema de decisão multicritério (WANG, 2010). Assim, este problema abrange a avaliação de especialistas no que tange aos vários critérios, qualitativos e quantitativos, necessários ao entendimento da situação, assim como requer métodos apropriados para a sua interpretação. (ALMEIDA, 2011)

A abordagem AMD visa fornecer aos gestores ferramentas que lhes permitam progredir na resolução de problemas de decisão, pelas quais vários objetivos, os quais frequentemente possuem pontos de vista contraditórios, devem ser levados em consideração, como é o caso, por exemplo, da dicotomia existente entre reduzir custos e aumentar a 
qualidade. (ALMEIDA, 2011)

\section{MODELAGEM MATEMÁTICA - MÉTODOS ORDINAIS}

O software WebPROA oferece soluções a partir dos três métodos ordinais Multicritério de Apoio à Decisão: o Método de Borda, de Condorcet e de Copeland.

Na Tabela 1, foram inseridas as alternativas de modelos de Drones e os critérios necessários nestes para a adequabilidade a Agricultura 4.0.

A modelagem matemática foi realizada pelo software WebPROA, desenvolvido pelo departamento de Engenharia de Produção da Universidade Sul Fluminense (UFF).

\subsection{LEVANTAMENTO DE DADOS}

Para o presente estudo de caso, selecionou-se oito principais modelos de Drones atuais no mercado. Essa seleção foi realizada através de pesquisas nos sites de fornecedores brasileiros.

Para as alternativas de Drones foram selecionados os seguintes modelos: Mavic 2 Pro; Inspire 2; Phantom 4 Pro; Batmap; Horus Maptop; Horus Verok; Inspire 2 X4S e Parrot Bluegrass.

Os critérios foram selecionados visando as principais características que um Drone deve possuir para ser utilizado na agricultura, como o alcance, tempo de voo, velocidade, resolução da câmera e o preço.

Na Tabela 1 a seguir, são apresentados os dados Alternativas vs. Critérios.

Tabela 1 - Alternativa vs. Critérios

\begin{tabular}{cccccc}
\hline Drones & Preço (R\$) & Alcance $(\mathbf{K m})$ & Tempo de voo (min) & $\begin{array}{c}\text { Velocidade (Km/ } \\
\text { h) }\end{array}$ & Resolução Cam (MP) \\
\hline Mavic 2 Pro & 11000 & 8 & 31 & 72 & 48 \\
Inspire 2 & 19000 & 7 & 27 & 94 & 20 \\
Phantom 4 Pro & 12000 & 7 & 30 & 50 & 20 \\
Batmap I & 50000 & 10 & 150 & 45 & 36 \\
Horus Maptor & 60000 & 10 & 60 & 80 & 20 \\
Horus Verok & 50400 & 10 & 120 & 80 & 24 \\
Inspire 2 X4S & 24000 & 7 & 27 & 94 & 20 \\
Parrot Bluegrass & 42000 & 2 & 25 & 40 & 16 \\
\hline
\end{tabular}

Fonte: Autor (2019)

A partir dos dados inseridos na tabela foram gerados resultados para os três métodos ordinais que serão detalhados nos tópicos a seguir.

\subsection{MÉTODO DE BORDA}

Segundo Kansas, Laukkanen e Kangas (2006), o método Borda é considerado um método de avaliação multicritério ordinal, sendo, em sua essência, a avaliação das alternativas que melhor se ajustem aos critérios definidos. Para uso deste método, o eleitor deve ordenar as alternativas de acordo com as suas preferências: a alternativa preferida recebe o maior ponto, a segunda alternativa recebe a segunda maior pontuação, e assim sucessivamente. Ao final, os pontos atribuídos pelos eleitores a cada alternativa são somados, e a alternativa que tiver obtido a maior pontuação é escolhida. Os autores também ressaltam que a qualidade no resultado do método dependerá da relevância dos critérios usados, sendo importante descartar aqueles considerados irrelevantes. Durante a aplicação deste método, todas as escolhas são feitas de forma voluntária, democrática e sem nenhuma indução. 
A partir dos dados inseridos na Tabela 1, os seguintes resultados representados na Tabela 2 a seguir, foram gerados por meio do método Borda.

Tabela 2 - Resultado para o método de Borda

\begin{tabular}{|c|c|c|c|c|c|c|}
\hline \multirow[b]{2}{*}{ Alternativas } & \multicolumn{5}{|c|}{ Critérios } & \multirow[b]{2}{*}{ Pontuação Total } \\
\hline & $\begin{array}{c}\text { Valor (RS) } \\
\text { MINIMIZAR }\end{array}$ & $\begin{array}{l}\text { Alcance (Km) } \\
M A X I M I Z A R\end{array}$ & $\begin{array}{c}\text { Tempo de vôo (min) } \\
M A X I M I Z A R\end{array}$ & $\begin{array}{c}\text { Velocidade } \mathbf{( K m} / \mathbf{h} \\
\text { MAXIMIZAR }\end{array}$ & $\begin{array}{c}\text { Resolução Câmera (MP) } \\
M A X I M I Z A R\end{array}$ & \\
\hline Mavic 2 Pro & 1 & 4 & 4 & 5 & 1 & 15 \\
\hline Inspire 2 & 3 & 6 & 6.5 & 1.5 & 5.5 & 22.5 \\
\hline Phantom 4 Pro & 2 & 6 & 5 & 6 & 5.5 & 24.5 \\
\hline Batmap & 6 & 2 & 1 & 7 & 2 & 18 \\
\hline Horus Maptor & 8 & 2 & 3 & 3.5 & 5.5 & 22.0 \\
\hline Horus Verok & 7 & 2 & 2 & 3.5 & 3 & 17.5 \\
\hline Inspire $2 \mathrm{X} 4 \mathrm{~S}$ & 4 & 6 & 6.5 & 1.5 & 5.5 & 23.5 \\
\hline Parrot Bluegrass & 5 & 8 & 8 & 8 & 8 & 37 \\
\hline
\end{tabular}

Fonte: Autor (2019)

No Método de Borda, atribui-se os primeiros lugares às alternativas com menor soma de scores de ranking. No caso em questão, o drone Mavic 2 Pro assumiu a primeira posição do ranking.

Posições:

$1^{\mathrm{a}}$ - Mavic 2 Pro;

$2^{\mathrm{a}}$ - Horus Verok;

$3^{\mathrm{a}}$ - Batmap;

$4^{\mathrm{a}}$ - Horus Maptor;

$5^{\mathrm{a}}$ - Inspire 2;

$6^{\mathrm{a}}$ - Inspire $2 \mathrm{X} 4 \mathrm{~S}$;

$7^{\mathrm{a}}$ - Phantom 4 Pro;

$8^{\mathrm{a}}$ - Parrot Bluegrass.

\subsection{MÉTOdo DE CONDORCET}

O método de Condorcet, considerado precursor da atual escola francesa de multicritério, trabalha com relações de superação. As alternativas são comparadas sempre duas a duas e constrói-se um grafo (Boaventura Neto, 2003) que expressa a relação entre elas. Através da representação da relação de preferência por um grafo, a determinação de alternativas dominantes e dominadas (quando existem) fica bastante facilitada. Quanto existe uma e só uma alternativa dominante, ela é a escolhida. Este método, menos simples, tem a vantagem de impedir distorções ao fazer com que a posição relativa de duas alternativas independa de suas posições relativas a qualquer outra. No entanto, pode conduzir ao chamado 'paradoxo de Condorcet', ou situação de intransitividade. Isso acontece quando a alternativa A supera a alternativa $B$, que supera a $C$, que por sua vez supera a alternativa $A$ ('Tripleta de Condorcet'), como apresentado na Figura 3 a seguir. Esta situação, embora possa ser aproveitada em certos problemas (Soares de Mello et al., 2004), impossibilita gerar uma ordenação das alternativas. Quando os ciclos de intransitividade não aparecem, o método de Condorcet deve ser preferido ao de Borda. (SOARES DE MELLO et al, 2004) 


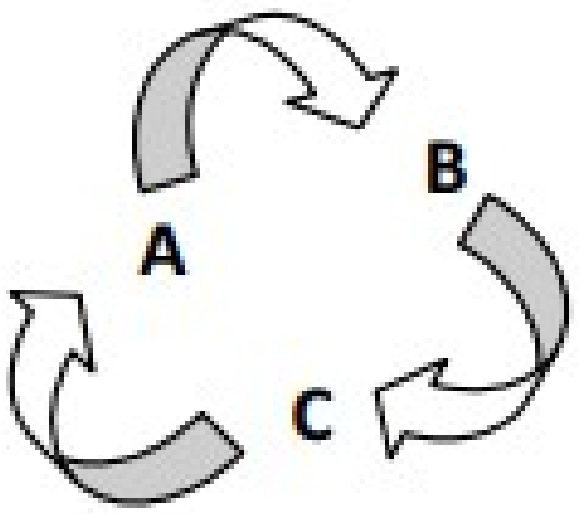

Fonte: Bana e Costa et al. (2013)

A partir dos dados da Tabela 1, os seguintes resultados representados na Tabela 3 a seguir, foram gerados pelo Método de Condorcet.

Tabela 3 - Resultado para o método de Condorcet

\begin{tabular}{|c|c|c|c|c|c|c|c|c|c|}
\hline Alternativas & $\begin{array}{c}\text { Mavic } \\
\text { 2 Pro }\end{array}$ & $\begin{array}{c}\text { Inspire } \\
\text { 2 }\end{array}$ & $\begin{array}{c}\text { Phantom } \\
\text { 4 Pro }\end{array}$ & Batmap & $\begin{array}{c}\text { Horus } \\
\text { Maptor }\end{array}$ & $\begin{array}{c}\text { Horus } \\
\text { Verok }\end{array}$ & $\begin{array}{c}\text { Inspire } \\
\text { 2 X4S }\end{array}$ & $\begin{array}{c}\text { Parrot } \\
\text { Bluegrass }\end{array}$ & Classificação \\
\hline Mavic 2 Pro & 0 & 1 & 1 & 1 & -1 & -1 & 1 & 1 & - \\
\hline Inspire 2 & -1 & 0 & -1 & -1 & 0 & -1 & 1 & 1 & - \\
\hline Phantom 4 Pro & -1 & 1 & 0 & -1 & -1 & -1 & 1 & 1 & - \\
\hline Batmap & -1 & 1 & 1 & 0 & 1 & 1 & 1 & 1 & - \\
\hline Horus Maptor & 1 & 0 & 1 & -1 & 0 & -1 & 0 & 1 & - \\
\hline Horus Verok & 1 & 1 & 1 & -1 & 1 & 0 & 1 & 1 & - \\
\hline Inspire 2 X4S & -1 & -1 & -1 & -1 & 0 & -1 & 0 & 1 & - \\
\hline Parrot Bluegrass & -1 & -1 & -1 & -1 & -1 & -1 & -1 & 0 & $\mathbf{8}^{\mathbf{o}}$ \\
\hline
\end{tabular}

Fonte: Autor (2019)

Para o presente estudo de caso, o software WebPROA não gerou um rankeamento dos Drones no método Condorcet pois o ciclo de intransitividade ocorreu. Isto significa que o método de Condorcet nem sempre induz uma pré-ordem no conjunto das alternativas.

\subsection{MÉTOdo DE COPELAND}

O método de Copeland apresenta como principal vantagem sempre fazer uma ordenação total, ao contrário do método de Condorcet, embora tenha sido originado deste (FERREIRA et al., 2011). Outra vantagem do método, segundo Gomes Jr. et al. (2004), é a redução da influência de alternativas irrelevantes.

O método usa a mesma matriz de adjacência que representa o grafo do método de Condorcet. Entretanto, ao contrário do método de Condorcet, o Copeland permite fazer uma ordenação total e manter a ordenação das alternativas que não pertencem ao ciclo de intransitividade.

A pontuação do Copeland para uma alternativa xj é determinada pela subtração do número de alternativas que a alternativa derrotou pelo número de alternativas no qual ela foi derrotada. Deste modo, sendo $P=\left\{P_{1}, \ldots, P_{n}\right\}$ a configuração das preferências, para um conjunto de alternativas $X$, o método pode ser dado da seguinte forma: $s\left(x_{j}, X, P\right)=\mid\{y \in X \mid$ $\left.x_{j} M_{y}\right\} \mid$ - $\left|\left\{y \in X \mid y M x_{j}\right\}\right|$, em que $x_{j} M_{y}\left(y M x_{j}\right.$ respectivamente) significa que a maioria dos eleitores preferem $\mathrm{x}_{\mathrm{j}}(\mathrm{y})$ a $\mathrm{y}\left(\mathrm{x}_{\mathrm{j}}\right)$. A função de escolha no Copeland, pode ser expressa por (NURMI, 1983): 


$$
F(X, P)=\{x \in X \mid s(x, X, P) \geq s(y, X, P), \forall y \in X\}
$$

A partir dos dados da Tabela 1, os seguintes resultados apresentados na Tabela 4 a seguir, foram gerados pelo Método de Copeland.

Tabela 4 - Resultado para o método de Copeland

\begin{tabular}{|c|c|c|c|c|c|c|c|c|c|}
\hline Alternativas & $\begin{array}{c}\text { Mavic } \\
\text { 2 Pro }\end{array}$ & $\begin{array}{c}\text { Inspire } \\
\text { 2 }\end{array}$ & $\begin{array}{c}\text { Phantom } \\
\text { 4 Pro }\end{array}$ & Batmap & $\begin{array}{c}\text { Horus } \\
\text { Maptor }\end{array}$ & $\begin{array}{c}\text { Horus } \\
\text { Verok }\end{array}$ & $\begin{array}{c}\text { Inspire } \\
\text { 2 X4S }\end{array}$ & $\begin{array}{c}\text { Parrot } \\
\text { Bluegrass }\end{array}$ & Pontuação Total \\
\hline Mavic 2 Pro & 0 & 1 & 1 & 1 & -1 & -1 & 1 & 1 & $\mathbf{3}$ \\
\hline Inspire 2 & -1 & 0 & -1 & -1 & 0 & -1 & 1 & 1 & $-\mathbf{2}$ \\
\hline Phantom 4 Pro & -1 & 1 & 0 & -1 & -1 & -1 & 1 & 1 & $\mathbf{- 1}$ \\
\hline Batmap & -1 & 1 & 1 & 0 & 1 & 1 & 1 & 1 & $\mathbf{5}$ \\
\hline Horus Maptor & 1 & 0 & 1 & -1 & 0 & -1 & 0 & 1 & $\mathbf{1}$ \\
\hline Horus Verok & 1 & 1 & 1 & -1 & 1 & 0 & 1 & 1 & $\mathbf{5}$ \\
\hline Inspire 2 X4S & -1 & -1 & -1 & -1 & 0 & -1 & 0 & 1 & $-\mathbf{4}$ \\
\hline Parrot Bluegrass & -1 & -1 & -1 & -1 & -1 & -1 & -1 & 0 & $-\mathbf{7}$ \\
\hline
\end{tabular}

Fonte: Autor (2019)

Este método gerou os seguintes resultados:

\section{Posições:}

$1^{\mathrm{a}}$ - empatados Batmap e Horus Verok;

$2^{\mathrm{a}}$ - Mavic 2 Pro;

$3^{\mathrm{a}}$ - Horus Maptor;

$4^{\text {a }}$ - Phantom 4 Pro;

$5^{\mathrm{a}}$ - Inspire 2;

$6^{\mathrm{a}}$ - Inspire $2 \mathrm{X} 4 \mathrm{~S}$;

$7^{\mathrm{a}}$ - Parrot Bluegrass.

\section{RESULTADOS E DISCUSSÕES}

Concluídas as modelagens dos métodos ordinais de Borda, Condorcet e Copeland, chegou-se às ordenações apresentadas na Tabela 5 a seguir.

Tabela 5 - Resultados dos métodos ordinais

\begin{tabular}{|c|c|c|}
\hline Borda & Condorcet & Copeland \\
\hline Mavic 2 Pro & & Batmap e Horus Verok; \\
Horus Verok & & Mavic 2 Pro \\
Batmap & Ciclo & Horus Maptor \\
Horus Maptor & de & Phantom 4 Pro \\
Inspire 2 & Intransitividade & Inspire 2 \\
Inspire 2 X4S & & Inspire 2 X4S \\
Phantom 4 Pro & & Parrot Bluegrass \\
Parrot Bluegrass & & \\
\hline
\end{tabular}

Fonte: Autor (2019)

Com o rankeamento realizado de acordo com seus respectivos métodos percebe-se que a informação quanto aos modelos de Drones e os critérios estabelecidos ficam mais claras e assim é possível para o decisor observar quais das alternativas se adequam melhor as suas necessidades e quais trarão resultados efetivos ao negócio. 


\section{SPOLM2O19}

XIX SIMPÓSIO DE PESQUISA OPERACIONAL E LOGISTICA DA MARINHA

A partir dos resultados gerados observa-se que tanto o modelo de Drone Mavic 2 Pro quanto o Horus Verok se adequam a Agricultura 4.0.

\section{CONSIDERAÇÕES FINAIS}

Esse trabalhou utilizou três metodologias ordinais de apoio a tomada de decisão para rankear modelos de Drones a serem utilizados na Agricultura 4.0. Os três métodos Borda, Condorcet e Copeland apresentaram resultados coerentes.

O trabalho atingiu o objeto de demonstrar que através das ferramentas e dos métodos de apoio a decisão é possível o decisor se nortear e realizar uma escolha mais assertiva, no contexto do estudo proposto, caberá ao decisor optar pela alternativa de Drone que atenderá a produção nas colheitas e a cultura específica plantada, dentro da capacidade financeira para tal investimento e conquistar uma agricultura sustentável de precisão.

A partir dos resultados gerados observa-se que tanto o modelo de Drone Mavic 2 Pro quanto o Horus Verok se adequam a Agricultura 4.0.

Salienta-se que a estrutura de apoio à tomada de decisão desenvolvida nessa pesquisa pode ser adaptada para atender a contextos organizacionais diversos, de acordo com os critérios estabelecidos e as alternativas disponíveis.

\section{REFERÊNCIAS BIBLIOGRÁFICAS}

AGRISHOW. Produção agrícola conectada com o universo digital: entenda a tendência da Agricultura 4.0. 2016. Disponível em: https://digital.agrishow.com.br/. Acesso em: 18 jan. 2019.

ALMEIDA, A. T. de (2011). O conhecimento e o uso de métodos multicritério de apoio a decisão. Recife: Editora Universitária da UFPE.

ANDRADE, R. de O. Drones sobre o campo - Avanços tecnológicos ampliam as possibilidades do uso de aeronaves não tripuladas na agricultura. Ed. 239. Janeiro, 2016. Disponível em: https://bdpi.usp.br/single.php? id=002784282. Acesso em 23 de mar de 2019.

ARENALES, M.; ARMENTANO, V.; MORABITO, R.; YANASSE, H. Pesquisa operacional. Rio de Janeiro: Elsevier, p.524, 2007.

BANA E COSTA, C.A.; ANGULO MEZA, L. \& OLIVEIRA, M.D. O Método MACBETH e Aplicação no Brasil. Engevista, no prelo 2013.

BITTENCOURT, M. e ANGULO-MEZA, L. WEBPROA, software livre para os métodos multicritérios à decisão borda, Condorcet e Copeland - in XLV SBPO, Natal - RJ, 2013

BOAVENTURA NETTO. P.O. Grafos: Teoria, Modelos, Algoritmos. Edgard Blücher, São Paulo, Editora Edgard Blücher, 2003.

EID, B. M.; CHEBIL, J.; ALBATSH, F.; FARIS, W. F. Challenges of Integrating

Unmanned Aerial Vehicles. In Civil Application. 5th International Conference on Mechatronics (ICOM’13) IOP Publishing. IOP Conf. Series: Materials Science and Engineering 53 (2013).

EMBRAPA. Agricultura, Sustentabilidade e Tecnologia. Brasília, DF, 2012. 34p.

Disponível em:

https://www.alice.cnptia.embrapa.br/bitstream/doc/1028545/1/AgriculturaSustentabilidadee Tecnologia.pdf. Acesso em: 25 fev. 2019. 
FERREIRA, R.O.; GOMES, F.P.; MELLO, J.C.C.B.S. Emprego de métodos ordinais multicritério na escolha de seleções cabeças de chave da copa do mundo de 2014. Relatórios de Pesquisa em Engenharia de Produção, v. 11, n. 11, 2011

GOMES, L. F. A. M., ARAYA, M. C. G. et CARIGNANO C. (2004), Tomada de Decisões em Cenários Complexos, Tradução de Marcela Cecilia González Araya, Pioneira Thomson Learning, São Paulo.

HONKAVAARA, E.; SAARI, H.; KAIVOSOJA, J.; PÖLÖNEN, I.; HAKALA, T.; LITKEY, P.; MÄKYNEN, J.; PESONEN, L. Processing and Assessment of Spectrometric, Stereoscopic Imagery Collected Using a Lightweight UAV Spectral Câmera for Precision Agriculture. Remote Sens. 2013, 5, 5006-5039.

INAMASU et al. Agricultura de Precisão, um novo olhar. Embrapa Instrumentação. Editora Cubo, 2011.

KANGAS, Annika; LAUKKANEN, Sanna; KANGAS, Jyrki. Social choice theory and its applications in sustainable forest management-a review. Forest Policy and Economics journal, v. 9, p. 77-92, 2006

MASSRUHÁ, S. M. F. S.; LEITE, M. A. de A.; MOURA, M. F. Os novos desafios e oportunidades das tecnologias da informação e da comunicação na agricultura (AgroTIC). In: MASSRUHÁ, S. M. F. S.; LEITE, M. A. de A.; LUCHIARI JUNIOR, A.; ROMANI, L. A. S. (Ed.). Tecnologias da informação e comunicação e suas relações com a agricultura. Brasília, DF: Embrapa, 2014. Cap. 1. p. 23-38.

MASSRUHÁ, S. M. F. S. Tecnologias da informação e da comunicação: o papel na agricultura. AgroANALYSIS: A Revista do Agronegócio da FGV, São Paulo, v. 35, n. 9, p. 29-31, 2015.

Ministério da Indústria, Comércio Exterior e Serviços. (2017). Projeto apoio aos diálogos setoriais união europeia - Brasil: MDIC. Mdic.gov.br. Disponível em: http://www.mdic.gov.br/images/publicacao DRONES-20161130-20012017-web.pdf. Acesso em 2 fev. 2019.

MONTEIRO, J. E. B. de A.; OLIVEIRA, A. F. de; NAKAI, A. M. TIC em agrometeorologia e mudanças climáticas. In: MASSRUHÁ, S. M. F. S.; LEITE, M. A. de A.; LUCHIARI JUNIOR, A.; ROMANI, L. A. S. (Ed.). Tecnologias da informação e comunicação e suas relações com a agricultura. Brasília, DF: Embrapa, 2014. Cap. 7. p. 121138.

Nurmi, H. (1983), Voting Procedures: A summary analysis. British Journal of Operational Research, vol. 82, pp. 522-539.

OLIVEIRA, T. P. A., PANTOJA, M. J., \& BRISOLA, M. V. (2016). Plano ABC: Contribuições Teóricas para o Novo Paradigma da Agropecuária e uma Proposta de Avaliação. Revista em Agronegócio e Meio Ambiente, 9(3), 719.

PAULO, SP. HAIMES, Y. Y. et CHANKONG, V. (1979), "Kuhn-Tucker Multipliers as Trade-Offs in Multiobjective Decision-Making Analysis”, Automática, Vol.15, pp.50-72, disponível em: http://www. scopus.com. Acesso em: 05 de jan. de 2019.

PEÑA-BARRAGÁN, J. M; et al. Object-based approach for crop row characterization in uav images for site-specific weed management. Institute for Sustainable Agriculture, IAS-CSIC, Córdoba (Spain). University of Califórnia, Berkeley, CA (USA). Proceedings of the 4th GEOBIA, May 7-9, 2012 - Rio de Janeiro - Brazil. p.426 
RODRIGUEZ, D. S. S, COSTA, H. G. et CARMO, L. F. R. R. S. do (2013), “Métodos de auxílio multicritério à decisão aplicados a problemas de PCP: Mapeamento da produção em periódicos publicados no Brasil”, Gest. Prod., Vol.20 No.1, pp134-146, disponível em: http://www.scielo.br/pdf/gp/v20n1/a10v20n1.pdf (Acesso: 11 de fevereiro de 2015).

SILVA NETO, M. Como utilizar imagens aéreas na agricultura de precisão? 13 de setembro de 2013. Disponível em: https://www.agrimensordofuturo.com. Acesso em: 26 jan. 2019.

SOARES DE MELLO, M.H.C.; QUINTELLA, H.L.M.M., SOARES DE MELLO, J.C.C.B. Avaliação do desempenho de alunos considerando classificações obtidas e opiniões dos docentes. Investigação Operacional Vol. 24, n. 2, p.187-196, 2004.

SWINTOM, S, M., LOWENBERG-DEBOER, J. Evaluating the profitability of site-specific farming, Journal of Production Agriculture, Madison, v.11, n.4, p.439-446, 1998.

VDMA VERLAG. Guideline Industrie 4.0r. 2016. Disponível em: https://industrie40.vdma.org/documents/4214230/5356229/VDMA+Guideline+Industrie+40. pdf/cd1e8ef0-626e-432d-a2da-5c7a990b1a9b . Acesso em: 4 jan. 2019.

WANG, W.-P. (2010). A fuzzy linguistic computing approach to supplier evaluation. Applied Mathematical Modelling, 34(10), 3130-3141. doi: 10.1016/j.apm.2010.02.002 